\title{
Interrelationship among physical activity, quality of life, clinical and sociodemographic characteristics in a sample of Nigerian patients with type 2 diabetes
}

\author{
Adeniyi, A.F., ${ }^{1}$ Ogwumike, O.O., ${ }^{1}$ Oguntola, D.A., ${ }^{1}$ Adeleye, J.O. \\ ${ }^{1}$ Department of Physiotherapy, College of Medicine, University of Ibadan, Ibadan, Nigeria \\ ${ }^{2}$ Department of Medicine, College of Medicine, University of Ibadan, Ibadan, Nigeria \\ Correspondence \\ Dr A.F. Adeniyi, Department of Physiotherapy, College of Medicine, University of Ibadan, Ibadan, Nigeria \\ •E-mail: adeniyifatai@yahoo.co.uk; fadeniyi@comui.edu,ng
}

\begin{abstract}
SUMMARY
A number of complexities surround the health and well-being of patients with type 2 diabetes. These difficulties relate to self-care efforts and outcomes, and several other factors play regulatory functions. This study was carried out to investigate the inter-relationship among physical activity, quality of life, and clinical and sociodemographic factors in a group of Nigerian patients with type 2 diabetes. The study was conducted at the outpatient clinics of two major healthcare facilities in Ibadan, Southwest Nigeria. Physical activity was assessed using the International Physical Activity Questionnaire, and quality of life was assessed using the Diabetes Quality of Life Brief Clinical Inventory. Clinical and sociodemographic characteristics were also documented. A total of 227 patients with type 2 diabetes with a mean age of $55.83 \pm 13.76$ years took part in the study. A total of $156(68.7 \%)$ of the participants, had low level of physical activity, while $81(35.7 \%)$ reported quality of life scores below the intermediate score and hypertension was the mo $63(27.8 \%)$ of the participants. Being 50 years and older $(\mathrm{OR}=2.5 ; 95 \% \mathrm{CI}=1.21-3.67)$ increased the odds of having lower quality of life, while physical activity of moderate-to-high intensity reduced the odds $(\mathrm{OR}=0.4 ; 95 \% \mathrm{CI}=0.2-0.8)$. A substantial proportion of patients with type 2 diabetes had below intermediate level quality of life, and most of them were physically inactive. Lower quality of life was also linked with sociodemographic and clinical variables but patients with moderate-to-high physical activity were likely to reduce by half the risk of poor quality of life.
\end{abstract}

KEY WORDS: physical activity, quality of life, sociodemographic characteristics

\section{INTRODUCTION}

Diabetes is one of the most important chronic diseases in the general population, when considering the impact on health (Wandell 2005). Type 2 diabetes is a chronic disease that affects the general health and well-being of a patient in various ways. For example, severe dietary restriction and daily self-administration of oral medications or insulin may adversely affect an individual's health-related quality of life (Redekop et al., 2002). Hence, the primary goals in treating patients with diabetes are maintaining blood glucose levels as close to normal as possible, and making a relatively normal quality of life achievable (Matthias et al., 2002).

The concept of quality of life is important to all patients and any intervention or group of interventions that does not improve the quality of life of the patient is not worth the effort. Based on this assertion, quality of life was characterized as "the ultimate goal of all health interventions" (Rubin and Peyrot 1999). Quality of life as a concept covers a broad range of human experience, and in the medical domain, it denominates aspects of health 
from the patient's or subject's point of view, and could better be expressed as "subjective health" or "functional status and wellbeing" (Wandell 2005). Clinicians and policymakers have recognized the importance of measuring health-related quality of life to inform patient management and policy decisions (Guyatt et al., 1993). The research field on quality of life has increased enormously since 1990.

Many factors affect the quality of life of patients with type 2 diabetes, and these include clinical and lifestyle issues. Diabetes affects health-related quality of life through macrovascular complications and associated non-vascular comorbidity, and also by adding to the total burden of disease (Wandell 2005). Future research areas should, therefore include transcultural and ethnic aspects and effects of lifestyle interventions (Wandell 2005). Significant associations have been demonstrated between socioeconomic status and health-related quality of life in the general population, but no significant association has been found between race and ethnicity and health-related quality of life among people with diabetes (Rubin and Peyrot 1999). Thus, knowledge concerning health-related quality of life in diabetic patients, as well as the determinants of the quality of life, is crucial (Wandell 2005).

A prominent lifestyle intervention for people with diabetes is the application of physical activity for the prevention and control of the disease. Although the use of physical activity has been well supported by literature, it is not well reported how this is linked with quality of life of people with diabetes. Efforts to prevent complications of diabetes often overlook the impact of the condition and its treatment on current quality of life (Bradley and Speight 2002). Coffey et al. (2002) reported that no study has been done to systematically quantify the degree to which type 1 and type 2 diabetes and their treatments, complications, and comorbidities affect quality of life. The authors further stated that empirical health utility scores were often not available in published economic analyses. Hence, this study was carried out to investigate the links among physical activity, quality of life, and clinical and sociodemographic characteristics in a group of Nigerian patients with type 2 diabetes.

\section{METHODOLOGY}

\section{Participants}

This study was carried out among 227 consecutively recruited patients with type 2 diabetes who were receiving medical care on outpatient basis at the diabetes clinics of the University College Hospital, Ibadan and State Hospital, Ring Road, Ibadan. This cross-sectional study recruited all consenting type 2 diabetes patients in the two hospitals who met the inclusion criteria. Participants were expected to be patients with type 2 diabetes whose duration of diagnosis was at least 6 months, and who could read and/or understand either Yoruba and/or English language. Patients with complications that may affect physical activity, such as blindness, amputation and other physical and cognitive disabilities were excluded from the study.

\section{Instrument}

\section{Sociodemographic data questionnaire}

Sociodemographic and clinical information on the participants, including age, gender, occupation, level of education, marital status and duration since diagnosis was obtained from the subjects using the sociodemographic data questionnaire. Other information included history of existing co-morbidities and complications.

\section{International Physical Activity Questionnaire (IPAQ)}

IPAQ was developed as an instrument for cross national monitoring of physical activity and inactivity across diverse countries and populations (Craig et al. 2003). IPAQ can be used to assess activities of different intensities and sedentary behaviours during work, transportation, and leisure time (Medina et al., 2013). It has two versions: the short version and the long version. The long version comprises 5 activity domains, asked independently while the short version comprises 4 activity domains. Both versions are recommended for use by either telephone or selfadministration methods (IPAQ guidelines, 2005).

The short version of IPAQ (IPAQ-SF) was used for this study. IPAQ-SF (7 items) was used to assess physical activity undertaken across a comprehensive set of domains including activities at work, as part of house and yard work, getting from place to place, and in spare time for recreation, exercise or sport. IPAQ-SF asks questions on three specific types of activity undertaken in the four domains (IPAQ Guidelines, 2005). The specific types of activities that were assessed, were walking, moderate intensity activities and vigorous intensity activities. IPAQ is a generic scale and has a reliability of 0.80 and criterion validity of 0.30 , which means that it is reliable, valid and of wide utility (Kurtze et al., 2008). 
The IPAQ-SF scoring protocol is such that the items in questionnaire are structured to provide separate scores on walking, moderate intensity and vigorous intensity activities. The total score for the short form requires summation of the duration (in minutes) and the frequency (days) of walking, moderate intensity and vigorous intensity activities.

Walking MET (minutes/ week) $=3.3 \mathrm{x}$ walking minutes $\mathrm{x}$ walking days.

Moderate MET (minutes/ week) $=4.0$ x moderate intensity activity minutes $\mathrm{x}$ moderate intensity days

Vigorous MET (minutes/week) $=8.0 \mathrm{x}$ vigorous intensity activity minutes $\mathrm{x}$ vigorous intensity days.

Total physical activity MET (minutes / week) = sum of walking + moderate + vigorous MET (minutes/ week) scores

$\mathrm{MET}=$ metabolic equivalent

Physical activity level was categorized into vigorous (with a minimum of $1500 \mathrm{MET}$ minutes/week), moderate (with at least 600 MET minutes / week), and low if activity is lower than $600 \mathrm{MET}$ minutes/ week (IPAQ guidelines, 2005).

\section{Diabetes Quality of Life Brief Clinical Inventory (DQOL-B)}

The DQOL-B is a shortened version of the original 60-item Diabetes Quality of Life Measure (DQOL) treatmentfocused instrument (Diabetes Control and Complications Trial Research Group (DCCT), 1988; Jacobson et al., 1994). It is used with persons with either Type 1 or 2 diabetes mellitus. The DQOL-B predicts self-reported diabetes care behaviours and satisfaction with diabetes control as effectively as the full-version DQOL. It was reported to be reliable $(\alpha=0.85)$ and valid during the initial development and validation study of 498 people (Burroughs et al., 2004). The DQOL-B is a 15-item inventory that yields a total score. The new total score correlates with the full DQOL scale and with the individual DQOL subscales. The items on the instrument cover a wide range of issues concerning people with diabetes that are addressable during routine clinical care. The scores of all items is summed up, giving a total scale score resulting in a grand measure score, which ranges from 15-75 with higher scores indicating better quality of life (Al-Maskari et al., 2011).

\section{Procedure}

Ethical approval of the University of Ibadan/ University College Hospital Ethics Review Committee and the consent of the physicians in charge of the patients were sought and obtained before commencing the study. An informed consent form stating the purpose of the study as well as assuring participants of confidentiality and anonymity was attached to the questionnaire. The questionnaires were translated into Yoruba for the benefit of Yoruba speaking participants who do not understand or speak English through a forward-back translation process. The translation was handled by a translator who is well versed in Yoruba and English languages. The questionnaires were selfadministered but assistance was available for those who needed help. The questionnaires were administered at the respective outpatient diabetes clinics of the selected hospitals. Sociodemographic and clinical information including age, sex, occupation, level of education, marital status and duration since diagnosis, was obtained from the participants. Others such as history of hypertension, and other co-morbidities and complications were included.

\section{Statistical Analysis}

Descriptive statistics of mean and standard deviation were used to analyse some of the data, while frequencies and percentages were used to summarize categorical data. Multivariate analysis was used to establish which patient characteristics were associated with quality of life scores below intermediate scores. Level of significance was set at $\mathrm{p} \leq 0.05$.

\section{RESULTS}

A total of 227 patients with type 2 diabetes consented to participate in this study and were subsequently enrolled. The mean age of the participants was $55.83 \pm 13.76$ years with 60-69 years being the modal age category (table 1). Table 1 shows that most of the participants $(62.2 \%)$ were diagnosed with diabetes less than 10 years before this study and hypertension was the most prevalent concomitant morbidity among the participants, reported by $63(27.8 \%)$ of the patients. Figure 1 presents the physical activity level of the participants. A total of 156 representing $68.7 \%$ of the participants presented with low levels of physical activity. The remaining $21.3 \%$ presented with moderate to high level of physical activity. 
Table 1. Clinical and sociodemographic characteristics of participants

\begin{tabular}{|c|c|}
\hline Variables & Frequency (n (\%) \\
\hline \multicolumn{2}{|l|}{ Age Category(years) } \\
\hline $30-39$ & $9(4.0)$ \\
\hline $40-49$ & $19(8.4)$ \\
\hline $50-59$ & $60(26.4)$ \\
\hline $60-69$ & $93(40.0)$ \\
\hline 70 and above & $46(20.3)$ \\
\hline \multicolumn{2}{|l|}{ Sex } \\
\hline Male & $58(25.6)$ \\
\hline Female & $169(74.4)$ \\
\hline \multicolumn{2}{|l|}{ Marital status } \\
\hline Married & $196(86.3)$ \\
\hline Single/Divorced & $31(13.7)$ \\
\hline \multicolumn{2}{|l|}{ Level of education } \\
\hline No formal education & $42(18.5)$ \\
\hline Primary & $38(16.7)$ \\
\hline Secondary & $56(24.7)$ \\
\hline Post-secondary & $91(40.1)$ \\
\hline \multicolumn{2}{|l|}{ Occupation } \\
\hline Unemployed & $23(10.1)$ \\
\hline Self-employed & $66(29.1)$ \\
\hline Civil servant & $86(37.9)$ \\
\hline Retiree & $52(22.9)$ \\
\hline \multicolumn{2}{|c|}{ Duration of Diagnosis (years) } \\
\hline $0-5$ years & $63(27.8)$ \\
\hline $6-10$ years & $78(34.4)$ \\
\hline$>10$ years & $86(37.9)$ \\
\hline \multicolumn{2}{|l|}{ Concomitant illness } \\
\hline Hypertension & $63(27.8)$ \\
\hline Osteoarthritis & $41(18.1)$ \\
\hline Prostate enlargement & $9(3.9)$ \\
\hline Mental disorder & $6(2.6)$ \\
\hline Obesity & $12(5.3)$ \\
\hline
\end{tabular}

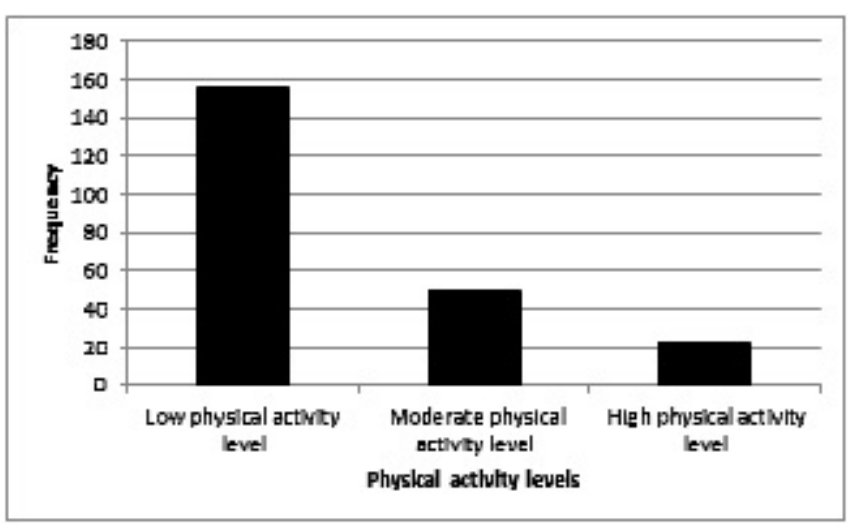

Figure 1. Distribution of physical activity levels of participants

The quality of life scores of the participants show that $81(35.7 \%)$ of them scored below the intermediate score, taken as 38. The results of the quality of life assessment of the participants is presented in table 2 . The table shows the frequencies of the highest confirmatory responses in respect of each of the summarized questions. A total of 163 $(71.8 \%)$ of the participants were concerned about absenteeism from work due to diabetes, 107 (47.1\%) were concerned about poor night sleep, 84 (37.0) reported daily activity limitation due to diabetes while 131 (57.7) had concerns about passing out as a result of diabetes.

Table 2. Frequency distribution of participants' response in confirmation of specific questions on quality of life items

\begin{tabular}{ll}
\hline Item/question & $\begin{array}{l}\text { Highest confirmatory } \\
\text { response }\end{array}$ \\
\hline Satisfaction with diabetes treatment & $156(68.7)$ \\
Satisfaction with time to manage diabetes & $159(70.0)$ \\
Eating to conceal diabetes status & $102(44.9)$ \\
Concern about absenteeism due to diabetes & $163(71.8)$ \\
Satisfaction with time to test sugar level & $156(68.7)$ \\
Satisfaction with time spent exercising & $154(67.8)$ \\
Poor night sleep because of diabetes & $107(47.1)$ \\
Satisfaction with sex life & $42(18.5)$ \\
Daily activity limitation due to diabetes & $84(37.0)$ \\
Frequent pain from needles and finger sticks & $178(78.4)$ \\
Burden on family due to diabetes & $37(16.3)$ \\
Frequently feeling ill & $49(21.6)$ \\
Concern about passing out due to diabetes & $131(57.7)$ \\
Satisfaction with time for checkups & $137(60.4)$ \\
Satisfaction with knowledge about diabetes & $82(36.1)$ \\
\hline
\end{tabular}

Table 3 contains the regression analysis for the risk of having below intermediate quality of life based on physical activity, sociodemographic and clinical variables. The table shows that participants who were 50 years and older had higher risk of having a lower quality of life compared to those who were younger $(\mathrm{OR}=2.5 ; 95 \% \mathrm{CI}=$ 1.21-3.67). It was also found that persons with physical activity of moderate to high intensity had less than half the odds of having reduced quality of life compared to those with low physical activity levels $(\mathrm{OR}=0.4 ; 95 \% \mathrm{CI}=$ $0.2-0.8)$. The existence of concomitant illness was found to present the highest odds of low quality of life. Compared to participants who did not report any apparent comorbidity, those who reported additional comorbidity had more than 3 times the odds of lower quality of life $(\mathrm{OR}=3.2 ; 95 \% \mathrm{CI}$ $=2.3-4.6)$. 
Table 3. Regression analyses showing factors associated with low quality of life by physical activity, clinical and sociodemographic characteristics

\begin{tabular}{ll}
\hline & $\begin{array}{l}\text { Below intermediate score } \\
(<\mathbf{4 5}) \\
\text { OR }(\mathbf{9 5 \%} \mathbf{C I})(\mathbf{n}=\mathbf{8 1})\end{array}$ \\
\hline Age (years) & 1 \\
$<50$ & $2.5(1.21-3.67)^{*}$ \\
$\geq 50$ & \\
Sex & 1 \\
Male & $1.4(0.8-2.1)$ \\
Female & \\
Marital status & 1 \\
Married & $1.7(1.1-2.6)^{*}$ \\
Single/Divorced & \\
Level of education & 1 \\
Educated & $1.2(0.8-1.8)$ \\
Not educated & \\
Occupation & 1 \\
Employed & $1.6(1.2-3.1)^{*}$ \\
Unemployed & \\
Physical activity & 1 \\
Low & \\
Moderate to high & \\
Duration of Diagnosis (years) & \\
$<$ 10 years & \\
$\geq 10$ years & $1.9(1.3-2.8)^{*}$ \\
Concomitant illness & \\
No & \\
Yes & \\
\hline & \\
\hline &
\end{tabular}

$\mathrm{OR}=$ Odds Ratio; $\mathrm{CI}=$ Confidence Interval

\section{DISCUSSION}

This study, carried out among patients with type 2 diabetes from outpatient clinics from the two largest hospitals in Ibadan, Southwest Nigeria, shows that about every 3 out of 4 patients with type 2 diabetes had low physical activity levels despite the fact that most of them had been diagnosed for over 5 years. This is not the first time such an observation will be made about people with diabetes in Nigeria. A previous study of patients with type 2 diabetes from two tertiary health institutions had reported similar findings (Adeniyi et al., 2010). The current finding shows that the situation is yet to improve noticeably in spite of public health education on the benefits of physical activity and health promotion. Another study also observed that low level of physical activity was one of the risk factors of type 2 diabetes among a group of Nigerians (Nyenwe et al.,
2003). A prevalence of physical inactivity of about $50 \%$ was also reported among persons living in a community in Northern Nigeria (Puepet and Ohwovoriole 2008).

Findings from the present study also show that a substantial amount of the participants reported low levels of quality of life. People with diabetes have been reported to have a worse quality of life than people with no chronic illness (Rubin and Peyrot, 1999). Another study by Glasgow et al. (1997) also documented that overall, respondents reported a moderate to low quality of life, relative to previous studies. The reason for the fairly low quality of life among patients with diabetes cannot be linked to a specific cause, as there are many reasons why a patient with type 2 diabetes will have low quality of life. However, in the general population, cross-sectional studies showed a consistently positive association between self-reported physical activity and health-related quality of life (Bize et al., 2007).

The fact that a sizeable number of the participants reported concerns about the components of their quality of life is also worthy of note. For example, a large number of the participants in this study strongly felt that they had to eat whatever they came in contact with because they did not want to disclose to people that they are living with diabetes. This is a form of manifestation of poor quality of life. It was reported that the greatest negative impact was observed for the domain 'Freedom to eat as I wish', indicating the strong influence of dietary restrictions on quality of life (Bradley and Speight 2002). Many of the participants were also concerned about absenteeism from work, poor night sleep, activity limitation and concerns about "passing out" among others. All these feelings confirmed the fact that the participants had low quality of life as a result of diabetes.

For those with low quality of life, it was discovered that age, marital status and occupation were the important sociodemographic variables that were linked to low quality of life. Being older than 50 years increased the risk of lower quality of life. Quality of life appears to be related to demographic, medical-history, and self-management factors (Glasgow et al., 1997; Wexler et al., 2006). Numerous demographic and psychosocial factors have been reported to influence quality of life, hence, such factors should be considered when handling patients (Rubin and Peyrot, 1999).

Additional comorbidities were found in this study to be associated with low quality of life. One of such comorbidities is mental health challenges. Findings 
demonstrate that anxiety, depressive symptoms, and neuropathy are prevalent in older adults with type 2 diabetes (Goldney et al., 2004; Chyun et al., 2006; Lin and Von Korff, 2008). The precise reason why type 2 diabetes individuals present with symptoms of mental health challenges is not well known but it may be due to a number of reasons including the complexity surrounding maintenance of good health while living with diabetes. Potentially important correlations had been demonstrated to link psychological factors, neuropathy, body mass index, and physical inactivity (Chyun et al., 2006).

It was observed that people with moderate to high physical activity had significantly reduced risk of low quality of life. A previous study had reported that the level of self-reported exercise was the only significant selfmanagement behaviour to predict quality of life, after controlling for demographic and medical variables (Glasgow et al., 1997). The reason why physical activity enhances quality of life may not be unconnected with the possibility of physical activity influencing many aspects of the life of patients, including the enhancement of glycaemic control in diabetic patients, which is at the very foundation of all the quality of life problems. In a previous study, it was reported that having better glycaemic control is associated with better quality of life (Rubun and Peyrot 1999). Improved glycaemic control in type 2 diabetes patients is associated with substantial short-term symptomatic, quality of life, and health economic benefits (Testa and Somonson 1998).

\section{CONCLUSIONS AND RECOMMENDATIONS}

In conclusion, the sample of Nigerian type 2 diabetes patients in this study had low physical activity level with more than a third having low to average quality of life. Their quality of life was influenced by sociodemographic characteristics, physical activity and clinical variables. Being older than 50 years, not married, unemployed, diagnosed for 10 years and above, and having concomitant illnesses increased the risk of low quality of life, while being physically active reduced the risk by half. Type 2 diabetes patients that fall into these categories should be accorded more attention in order to provide care that will not only improve glycaemic control but also quality of life. In addition, physical activity should be emphasized as an important component of the care of people with type 2 diabetes.

\section{References}

Adeniyi A.F., Fasanmade A.A., Aiyegbusi O.S. and Uloko A.E. 2010. Physical activity levels of type 2 diabetes patients seen at the outpatient diabetes clinics of two tertiary health institutions in Nigeria. Nigerian Quarterly Journal of Hospital Medicine 20(4): 165-170.

Al-Maskari M.Y., Al-Shookri A.O., Al-Adawi S.H. and Lin, K.G. 2011. Assessment of quality of life in patients with type 2 diabetes mellitus in Oman. Saudi Medical Journal 32(12): 1285-1290.

Bize R., Johnson J.A. and Plotnikoff R.C. 2007. Physical activity level and health-related quality of life in the general adult population: A systematic review. Preventive Medicine 45(6): 401-415.

Bradley C. and Speight J. 2002. Patient perceptions of diabetes and diabetes therapy: assessing quality of life. Diabetes/Metabolism Research and Reviews 18(3): S64-S69.

Burroughs T.E., Desikan R., Waterman B.M., Gilin D. and McGill, J. 2004. Development and validation of the diabetes quality of life brief clinical inventory. Diabetes Spectrum 17(1): 41-49.

Chyun D.A., Melkus G.D., Katten D.M., Price W.J., Davey J.A., Grey N., Heller G. and Wackers F.J.T. 2006. The association of psychological factors, physical activity, neuropathy, and quality of life in type 2 diabetes. Biological Research for Nursing 7(4): 279-288.

Coffey J.T., Brandle M., Zhou H., Marriott D., Burke R., Tabaei B.P., Engelgau M.M., Kaplan R.M. and Herman W.H. 2002. Valuing health-related quality of life in diabetes. Diabetes Care 25(12): 2238-2243.

Craig C.L., Marshall A.L., Sjostrom M., Bauman A.E., Booth M.L., Ainsworth B.E., Pratt M., Ekelund U., Yngve A. and Sallis, J.F. 2003. International Physical Activity Questionnaire: 12-Country Reliability and Validity. Medicine and Science in Sports and Exercise 35(8): 1381-95

Diabetes Control and Complications Trial Research Group. 1988. Reliability and validity of a diabetes quality-of-life measure for the Diabetes Control and Complications Trial (DCCT). Diabetes Care 11(9): 725-732.

Glasgow R.E., Ruggiero L., Eakin E.G., Dryfoos J. and Chobanian L. 1997. Quality of life and associated characteristics in a large national sample of adults with diabetes. Diabetes Care 20(4): 562-567.

Goldney R.D., Phillips P.J., Fisher L.J. and Wilson D.H. 2004. Diabetes, depression, and quality of Life: A population study. Diabetes Care 27(5): 1066-1070.

Guyatt G.H., Feeny D.H. and Patrick D.L. 1993. Measuring health-related quality of life. Annals of Internal Medicine 118(8): 622-629.

International Physical Activity Questionnaire Guidelines. 2005. http://www.ipaq.ki.se accessed 18/10/2015 $12.22 \mathrm{pm}$ 
Jacobson A.M., de Groot M., Samson J.A. 1994. The evaluation of two measures of quality of life in patients with type I and type II diabetes. Diabetes Care 17(4): 267-274.

Kurtze N., Rangul V. and Hustvedt B.E. 2008. Reliability and validity of the international physical activity questionnaire in the Nord-Trøndelag health study (HUNT) population of men. BMC Medical Research Methodology 8: 63.

DOI: 10.1186/1471-2288-8-63.

Lin E.H.B. and Von Korff M on behalf of the WHO WMH Survey Consortium. 2008. Mental disorders among persons with diabetes-Results from the World Mental Health Surveys. Journal of Psychosomatic Research 65(6): 571-580.

Matthias R., Fliege H., Hildebrandt M., Schirop T. and Klapp B.F. 2002. The network of psychological variables in patients with diabetes and their importance for quality of life and metabolic control. Diabetes Care 25(1): 35-42.

Medina C., Barquera S. and Janssen I. 2013. Validity and reliability of the International Physical Activity Questionnaire among adults in Mexico. Revista Panamericana de Salud Pública 34(1): 21-28.

Nyenwe E.A., Odia O.J., Ihekwaba A.E., Ojule A. and Babatunde S. 2003. Type 2 diabetes in adult Nigerians: a study of its prevalence and risk factors in Port Harcourt, Nigeria. Diabetes Research and Clinical Practice 62 (3): 177-185.
Puepet F.H. and Ohwovoriole A.E. 2008. Prevalence of risk factors for diabetes mellitus in a non-diabetic population in Jos, Nigeria. Nigerian Journal of Medicine 17(1): 71-74.

Redekop W.K., Koopmanschap M.A., Stolk R.P., Rutten G.E.H.M., Wolffenbuttel B.H.R. and Niessen L.W. 2002. Health-related quality of life and treatment satisfaction in Dutch patients with type 2 diabetes. Diabetes Care 25(3): 458463.

Rubin R.R. and Peyrot M. Quality of life and diabetes. Diabetes/Metabolism Research and Review 1999; 15: 205-218.

Testa M.A. and Simonson D.C. Health economic benefits and quality of life during improved glycemic control in patients with type 2 diabetes mellitus: A randomized, controlled, double-blind trial. Journal of American Medical Association 1998; 280(17): 1490-1496.

Wändell P.E. 2005. Quality of life of patients with diabetes mellitus: An overview of research in primary health care in the Nordic countries. Scandinavian Journal of Primary Health Care 23(2): 68-74.

Wexler D.J., Grant R.W., Wittenberg E., Bosch J.L., Cagliero E., Delahanty L., Blais M.A. and Meigs J.B. 2006. Correlates of health-related quality of life in type 2 diabetes. Diabetologia 49(7): 1489-1497. 